\title{
The effects of septal lesions or scopolamine injections on retention of habituation to a novel environment
}

\author{
ELIZABETH WORSHAM and LEONARD W. HAMILTON \\ Rutgers University, New Brunswich, New Jersey 08903
}

\begin{abstract}
Previous studies have shown that scopolamine injections or medial septal lesions disrupt the habituation of an exploratory head-poke response. The present study was designed to extend these. results and to determine the effects of these manipulations upon the retention of the habituated response. Exploratory responding was assessed in two 50 -min sessions separated by 10 days. The results suggested an impairment of habituation following either scopolamine injections or septal lesions during both Phases 1 and 2. Septal lesions produced similar effects regardless of prior experience in the apparatus; however, injections of scopolamine resulted in different effects upon performance as a function of prior experience. Thus in contrast to previous suggestions that the effects of these two manipulations on habituation were identical, the present study suggests that there are differences which can be observed under the appropriate testing conditions.
\end{abstract}

Habituation is frequently used as a model of inhibition, i.e., in the course of exploring the environment, an animal must learn which responses are relevant to reward and which responses lead to nonreward. These latter responses are inhibited.

Carlton (1969) has postulated the existence of a central cholinergic system which controls behavioral inhibition. Other studies (e.g., Hamilton \& Grossman, 1969) have shown that the system defined by Carlton may be represented, at least in part, within the limbic system.

A task that measures exploratory responses in a novel environment has been designed to measure habituation. In this task, normal adult rats initially show high rates of performing a discrete exploratory response (head-poking into a tube) which gradually decreases with continued exposure to the novel situation, while the mean duration per response increases (Feigley \& Hamilton, 1971; Feigley, Parson, Hamilton, \& Spear, 1972). These studies found this task to be sensitive to the functional integrity of cholinergic mechanisms. The effect of both medial septal lesions and scopolamine injections resulted in no change from control animals in the number of exploratory responses during the test period, but changes were found in response duration. Response duration of control rats increased with increased exposure to the novel situation, while the response duration of the septal and scopolamine groups remained constant and unchanging over time as if no

This research was supported by grants from the National Institute of Mental Health (MH 16448) and the Rutgers Research Council to L. W. Hamilton and was completed during the tenure of an NDEA Title IV Fellowship to E. Worsham. Requests for reprints should be sent to Leonard W. Hamilton. Department of Psychology, Rutgers University, New Brunswich, N. J. 08903. habituation was occurring (Feigley \& Hamilton, 1971).

The present study used the same response measure, head-poking in a novel chamber, to assess the effects of septal lesions and central cholinergic blockade on the retention of habiutation over a 10-day period.

\section{METHOD}

\section{Subjects}

The subjects were 62 male albino rats obtained from Carworth Company (CFN strain), weighing approximately $200-250 \mathrm{~g}$ at the start of the experiment. They were individually housed under constant illumination with ad-lib access to food and water.

\section{Apparatus}

Three identical Plexiglas chambers $(20 \times 21 \times 21 \mathrm{~cm})$ with wire mesh floors were used (see Feigley \& Hamilton, 1971 for details). A single tube $(12 \times 5 \mathrm{~cm} \mathrm{diam})$ centered $5.5 \mathrm{~cm}$ above the floor contained an infrared light beam which operated an electronic switch when it was interrupted.

\section{Procedures}

Surgery and Histology. Surgery was performed under Equithesin anesthesia. Septal lesions were produced by passing $1.5 \mathrm{~mA}$ of anodal current for $15 \mathrm{sec}$ through a single stainless steel electrode $15 \mathrm{deg}$ from the vertical and lowered stereotactically to the following de Groot (1959) coordinates: $A=7.6, H=1.0$, and $L=.5 \mathrm{~mm}$ across the midline. The operated controls received the same treatment except that electrode was not lowered into the brain.

Following behavioral testing, histological verification of the lesion sites were completed by staining every fifth 60 -micra frozen section with cresyl violet and examining the tissue microscopically.

\section{Testing}

Prior to surgery, the 62 rats were divided into the following groups:

Group Con-Con: A total of 18 rats, comprised of seven rats that were tested during both phases as normal controls, six rats that were tested during both phases as operated controls, and five rats that were tested during both phases following injections of saline.

Group Sep-Sep. A group of 13 rats that received septal lesions 10 days prior to Phase 1 .

Group Scop-Scop: A group of 10 rats that received injections of 
$1.0 \mathrm{mg} / \mathrm{kg}$ of scopolamine hydrobromide $20 \mathrm{~min}$ prior to both Phase 1 and Phase 2.

Group C-Sep: A group of 11 rats that received septal lesions the day after being tested on Phase 1 as controls.

Group C-Scop: A group of 10 rats that received injections of $1.0 \mathrm{mg} / \mathrm{kg}$ of scopolamine hydrobromide $20 \mathrm{~min}$ prior to Phase 2 after being tested on Phase 1 as controls.

For statistical purposes, and for clarity of graphical presentation, the groups that were essentially untreated in Phase 1 were pooled to form a group called Comb-Con: A control group of 39 rats, consisting of the 18 rats from Group Con-Con plus the 21 rats from Groups C-Scop and C-Sep during Phase 1 (i.e., prior to the administration of experimental treatments).

On the day of Phase 1 testing, each animal was placed into the apparatus for a 50 -min session. The frequency and total duration of head pokes in the tube were recorded at 5-min intervals. Phase 2 testing was conducted 10 days later using exactly the same procedure.

\section{Statistical Analyses}

All data were analyzed by means of a two-factor (repeated measures, unweighted means) analysis of variance (Winer, 1962). When a significant main effect was obtained, individual comparisons were carried out using the Fisher test for the least significant difference (Federer, 1955) to yield critical values for comparing group means.

\section{RESULTS AND DISCUSSION}

\section{Histological}

The lesions varied only slightly in size and location, resulting in extensive damage to the medial septum and the diagonal band, with slight damage to the lateral septum on the side through which the angled electrode was lowered. The tract of the diagonal band was damaged dorsal to the level of the anterior commissure, and there was virtually no tissue destruction ventral to the level of the anterior commissure. The lesions extended posteriorly to the columns of the fornix.

\section{Phase 1}

The rate and mean duration of exploratory responding of the control rats during Phase 1 was consistent with the previous results of Feigley and Hamilton (1971) and Feigley et al. (1972).

Rats which received septal lesions prior to Phase 1 (Group Sep-Sep) made fewer exploratory head pokes than rats in either Group Comb-Con or Group Scop-Scop, $F(2,59)=3.43$, $p<.05$. The number of pokes emitted over intervals was significant, $F(9,531)=17.4, p<.001$, with all groups decreasing their response frequency over sessions (see Figure 1A). When these same groups were compared on mean poke duration, Group Sep-Sep and Group Scop-Scop were significantly different from controls, $F(2,59)=13.4, p<.01$. The Combined Cons had an increasing poke duration across intervals, whereas the other two groups' response duration remained at a low level and were not significantly different from each other (see Figure 1B).

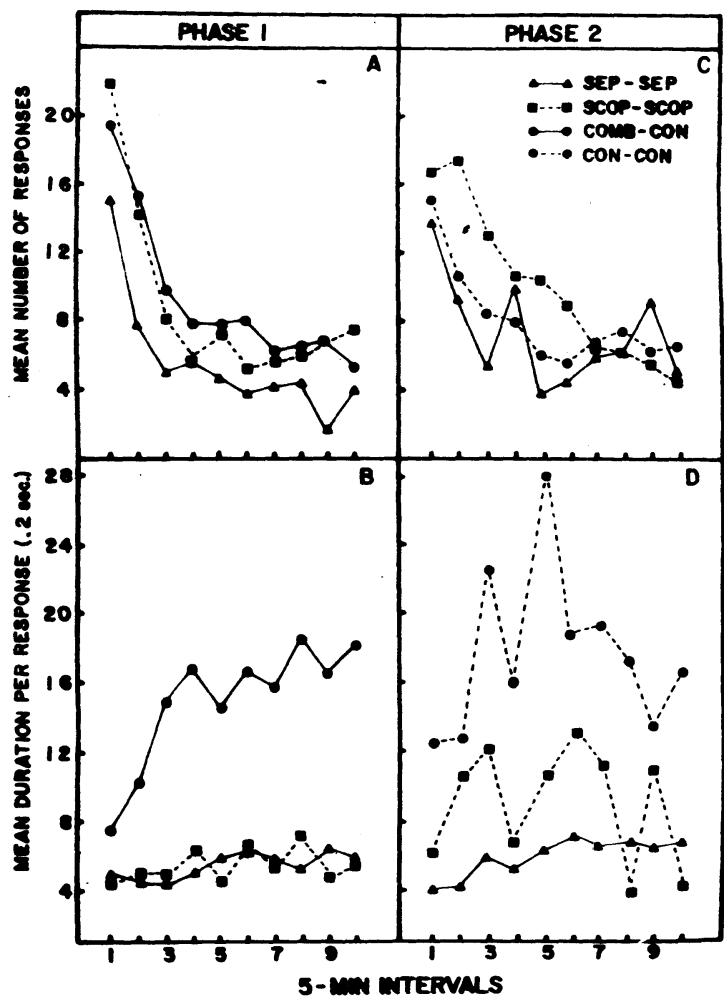

Figure 1. Response measures showing the effects of scopolamine injections and septal lesions when the groups had the same treatment conditions during both phases. Panels $A$ and $C$ represent the response frequency as a function of intervals. Panebs $B$ and D represent the response duration of the groups over intervals.

Thus, as in previous experiments (Feigley \& Hamilton, 1971), both scopolamine and septal lesions resulted in a disruption of the normal pattern of exploratory habituation.

\section{Phase 2}

Normal rats showed virtually complete spontaneous recovery when tested 10 days later. There was again a decline in response rate over time and an increase in mean poke duration over time. This complete lack of effect of prior experience was somewhat surprising, especially in view of the rapid within-sessions effects. Data collected more recently indicate that this spontaneous recovery is complete within $24 \mathrm{~h}$, suggesting a very transient effect on behavior under normal conditions.

There were no differences among Groups Sep-Sep, Scop-Scop, and Con-Con in the number of pokes during Phase $2(F<1)$, but the number of pokes over intervals was again significant, $F(9,324)=11.85$, $\mathrm{p}<.01$, with all three groups decreasing their rate over intervals (see Figure 1C). However, there was a significant groups effect on mean poke duration, $F(2.33)=5.62, p<.01$. All three groups were different, with the rats in Group Sep-Sep having the lowest mean poke duration, while Group Scop-Scop 


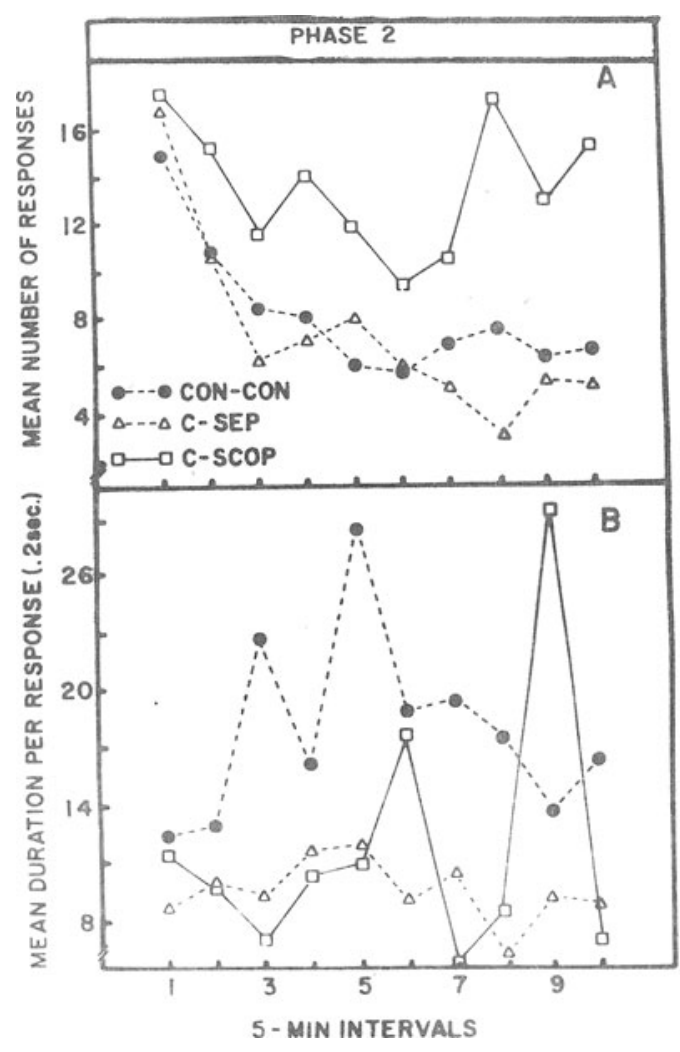

Figure 2. Response measures showing the effects of scopolamine injections and septal lesions during Phase 2 when all groups had been run as controls during Phase 1. Panel $A$ represents the response frequency as a function of intervals. Panel $B$ represents the response duration of the groups over intervals.

was intermediate and quite variable across intervals.

Rats which received scopolamine prior to Phase 2 only (Group C-Scop) poked significantly more than either the Groups Con-Con or C-Sep, $F(2,33)=4.11$, $p<.05$. There were no significant differences between these three groups on mean poke duration. However, this lack of significant difference was probably due to the increase in variability of poke duration as habituation progressed, so a separate analysis of variance was done on the first three 5-min intervals within the session, since it appeared that there might be differences in habituation early in the session. A significant groups effect was obtained, $F(2,36)=3.7, p<.05$. The controls had significantly greater mean poke duration than either of the other two groups which were not different from each other (see Figure 2).

Thus, septal lesions produced similar effects regardless of prior experience in the apparatus. Therefore, even if the rats had shown habituation to the chamber as controls during Phase 1 (Group C-Sep), they exhibited a decrease in mean poke duration in Phase 2 as if they had no prior exposure to the chamber. Even if septal lesions were produced prior to Phase 1 , the 2 nd test day did not reflect the effects of prior exposure.
In contrast, injections of scopolamine resulted in markedly different effects on performance as a function of prior experience. When the rats had been tested in Phase 1 with scopolamine, the rate of responding was somewhat higher in Phase 2 , and the increase in response duration and variability over the 5-min intervals was indicative that some habituation was occurring. These data support the suggestion of Feigley and Hamilton (1971) that the onset and duration of exploratory responding may be under separate neural control. Scopolamine injections only prior to Phase 2 (Group C-Scop) result in a rather dramatic increase in rate of responding not usually seen in this apparatus. The clear implication is that the prior experience under control conditions had an effect on the rats. At the present time, we have no clear explanation of this effect, although it could be related to a stimulus dissociation phenomenon.

The pattern of results suggest that the neural systems disrupted by septal lesions may be rather different from those disrupted by scopolamine injections, especially with respect to possible changes in memory processes. These data suggest the interesting possibility that scopolamine may effect changes in both primary habituation processes and some sort of memory deficit since a strict habituation interpretation cannot account for the increase in response duration (Phase 2), nor can it account for the increased rate of responding of Group C-Scop in Phase 2.

Previous data have suggested that the results of these two manipulations on habiutation were identical. However, the present experiment suggesis that there are both qualitative and quantitative differences which can be observed in the appropriate testing conditions.

\section{REFERENCES}

Carlton, P. L. Brain-acetylcholine and inhibition. In J. T. Tapp (Ed.), Reinforcement and behavior. New York: Academic Press, 1969.

DEGROOT, J. The rat forebrain in stereotaxic coordinates. Amsterdam: N. V. Noorde-Hollanshe Uitgevers Maatschappi, 1959.

FEDERER, W. T. Experimental design. New York: MacMillan, 1955.

Feigley, D. A., \& Hamilton, L. W. Response to novel environment following septal lesions or cholinergic blockade in rats. Journal of Comparative and Physiological Psychology, 1971, 76, 496-504.

Feigley, Parson, Hamilton, \& Spear. Development of habituation to novel environments in the rat. Journal of Comparative and Physiological Psychology, 1972. 79, 443-452.

Hamilton, L. W., \& Grossman, S. P. Behavioral changes following disruption of central cholinergic pathways. Journal of Comparative and Physiological Psychology, 1969, 69, 76-82.

WINER, B. J. Statistical principles in experimental design. New York: McGraw-Hill, 1962.

(Received for publication October 26, 1975.) 